\title{
IRP06
}

\section{Experimental Determination of Hydrate Phase} Equilibrium

Ehsan Kamari* (Research institute of Petroleum Industry(RIPI)) \& M. Oyarhossein (Research institute of Petroleum Industry (RIPI))

\section{SUMMARY}

Iran's proved natural gas reserves are the world's second largest reserves. Mainly, because of different climate changes and different reservoirs' characterizations, studying the behavior of producing outcome fluids and their transportation is greatly essential. One of the main problems occurs in the gas reservoirs is related to the hydrate formation while producing from a well, either in production strings or production lines (before and after choke).

Effective parameters which lead to form hydrates are: high pressure, low temperature and water presence; and therefore, the high possibility of having this phenomenon in Iranian reservoirs is quite obvious especially in the cold climates and for the gas wells. Hydrate formation in production lines and equipments will also result in many different obstacles such as: complete or partial closure in production lines and heat exchangers, erosion of the equipment, pressure reduction, etc. In this paper, by the experimental data from one the Iranian sour gas field, the conditions of hydrate formation, which will be helpful to determine the safe/unsafe zones by P-T curves, will be discussed. In addition, the final data will be compared to other correlations, as well. 
EAGE 